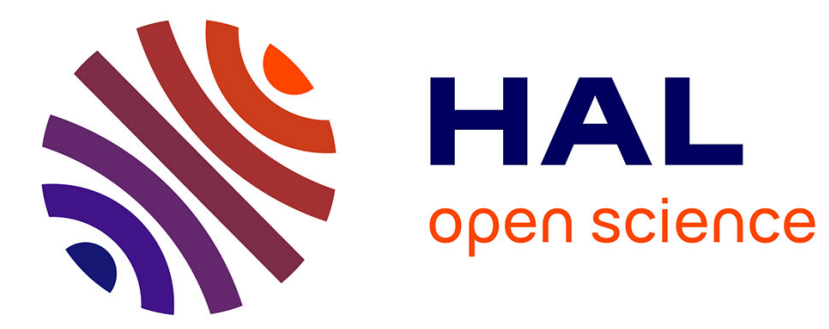

\title{
Gaze leading is associated with liking
}

Ouriel Grynszpan, Jean-Claude Martin, Philippe Fossati

\section{To cite this version:}

Ouriel Grynszpan, Jean-Claude Martin, Philippe Fossati. Gaze leading is associated with liking. Acta Psychologica, 2017, 173, pp.66 - 72. 10.1016/j.actpsy.2016.12.006 . hal-01426940

\section{HAL Id: hal-01426940 https://hal.sorbonne-universite.fr/hal-01426940}

Submitted on 5 Jan 2017

HAL is a multi-disciplinary open access archive for the deposit and dissemination of scientific research documents, whether they are published or not. The documents may come from teaching and research institutions in France or abroad, or from public or private research centers.
L'archive ouverte pluridisciplinaire HAL, est destinée au dépôt et à la diffusion de documents scientifiques de niveau recherche, publiés ou non, émanant des établissements d'enseignement et de recherche français ou étrangers, des laboratoires publics ou privés. 
Running head: Gaze leading is associated with liking

\section{Gaze leading is associated with liking}

Ouriel Grynszpan ${ }^{a}$, Jean-Claude Martin ${ }^{\mathrm{b}}$ and Philippe Fossatic

${ }^{a}$ Institut des Systèmes Intelligents et de Robotique (ISIR), Université Pierre et Marie Curie, CNRS, Paris, France, ouriel.grynszpan@upmc.fr

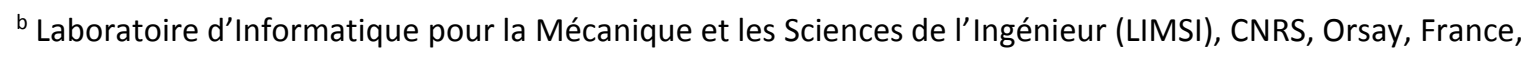
martin@limsi.fr

' Inserm U 1127, CNRS UMR 7225, Sorbonne Universités, UPMC Univ Paris 06, UMR S 1127, Institut du Cerveau et de la Moelle, ICM-A-IHU, Social and Affective Neuroscience (SAN) Laboratory \& Prisme Platform, Paris, France, philippe.fossati@upmc.fr

* Correspondence: Ouriel Grynszpan, ISIR (Institut des Systèmes Intelligents et de Robotique), Université Pierre et Marie Curie, Pyramide - T55/65, CC 173 - 4 place Jussieu, 75005 Paris, France, Tel : +33 (0) 144276369 , ouriel.grynszpan@upmc.fr

Reference of the article: Grynszpan, O., Martin, J.-C., \& Fossati, P. (2017). Gaze leading is associated with liking. Acta Psychologica, 173, 66-72. https://doi.org/10.1016/j.actpsy.2016.12.006 


\section{Gaze leading is associated with liking}

Abstract: Gaze plays a pivotal role in human communication, especially for coordinating attention. The ability to guide the gaze orientation of others forms the backbone of joint attention. Recent research has raised the possibility that gaze following behaviors could induce liking. The present study seeks to investigate this hypothesis. We designed two physically different human avatars that could follow the gaze of users via eye-tracking technology. In a preliminary experiment, 20 participants assessed the baseline appeal of the two avatars and confirmed that the avatars differed in this respect. In the main experiment, we compared how 19 participants rated the two avatars in terms of pleasantness, trustworthiness and closeness when the avatars were following their gaze versus when the avatar generated gaze movements autonomously. Although the same avatar as in the preliminary experiment was rated more favorably, the pleasantness attributed to the two avatars increased when they followed the gaze of the participants. This outcome provides evidence that gaze following fosters liking independently of the baseline appeal of the individual.

Keywords: social interactions; joint attention; chameleon effect; eye-tracking; gaze-contingent display; avatar 


\section{Introduction}

Social interactions involve a diversity of non-verbal behaviors. Gaze, in particular, plays a pivotal role in our daily social experiences. Eyes are commonly regarded as the "window of the soul" (BaronCohen, Wheelwright, \& Jolliffe, 1997; Hoekstra, Prendinger, Bee, Heylen, \& Ishizuka, 2007). Infants start demonstrating visual preference for the eye region as early as 3 months of age (Haith, Bergman, \& Moore, 1977). As demonstrated by Kobayashi and Kohshima (2001) who compared the morphology of eyes in half of the known primate species, the human eye holds specific features that are exceptionally well suited for displaying one's orientation of gaze: highly contrasting white sclera and dark iris; large ratio of exposed sclera; outline elongated in the horizontal direction. These specific features are believed to stem from an evolutionary adaptation that optimized the social use of gaze in human communication. The direction of gaze acts as a major indicator of one's focus of attention and thus provides guidance in the interpretation of one's intention (Baron-Cohen, 1997). It is instrumental in our ability to attribute mental states to others. Lacking skills in interpreting the mental significance of the eyes is profoundly disabling and has been linked to the Autism Spectrum Disorder (Baron-Cohen, Campbell, Karmiloff-Smith, Grant, \& Walker, 1995).

Social interactions between human beings relies on their ability to coordinate attention. This fundamental human capacity rests on what has been termed "joint attention" (Mundy \& Newell, 2007). Joint attention refers to various forms of reciprocal social behaviors involving gaze, pointing gestures, deictic verbal expressions or a combination of those, that are used for the purpose of enabling two or more individuals to focus on a common point of interest. It develops during early infancy and starts appearing when the baby is about six months old (Charman, 2003; Scaife \& Bruner, 1975). The ability to follow the gaze direction of others forms the backbone of joint attention (Emery, 2000). This ability has been designated as "gaze leading" or "gaze following" depending on which side is being observed, that is, the person whose gaze is being followed or, respectively, the person following the gaze of another individual. Mundy and Newell (2007) drew a distinction between 
responding to joint attention and initiating joint attention. Recent brain imaging studies brought support to this distinction by showing that responding to and initiating joint attention recruited different brain networks (Oberwelland et al., 2016; Redcay, Kleiner, \& Saxe, 2012; Schilbach et al., 2010). Responding to joint attention has been extensively studied using an adaptation of the classical Posner paradigm (Posner, 1980) where participants, who are shown a human face with eyes moving in a given direction, exhibit an reflexive reaction of orienting their attention in the same direction (Driver, 1999; see reviews in Frischen, Bayliss, \& Tipper, 2007; Lachat, Conty, Hugueville, \& George, 2012). By contrast, initiating joint attention has received lesser attention and is only recently beginning to be approached experimentally (Bayliss et al., 2013; Schilbach et al., 2010). The present study sought to investigate the link between initiating joint attention by leading the gaze of another person in a face-to-face situation and the degree of liking for this person.

To operationalize the study of gaze leading in laboratory settings, experimenters need to expose participants to a human face that follows their gaze. This has been made possible with the advent of virtual avatars and advanced eye-tracking technology. Eye-trackers have been used to create virtual agents that adapt to the user's gaze in real-time (Bailly, Raidt, \& Elisei, 2010; Hoekstra et al., 2007; Peters, Asteriadis, \& Karpouzis, 2010; Wang, Chignell, \& Ishizuka, 2006). Recent research projects have been specifically devoted to the design of virtual avatars that can follow the gaze of users in real-time via eye-tracking technology (Courgeon, Rautureau, Martin, \& Grynszpan, 2014; Kim \& Mundy, 2012; Wilms et al., 2010). These gaze following avatars enable more controlled and systematic experiments than what a human performer could achieve. They have been instrumental in research on gaze leading. For instance, they were used to identify cortical regions involved in leading the gaze of others (Oberwelland et al., 2016; Schilbach et al., 2010), to examine the influence of gaze leading on memory of faces (Kim \& Mundy, 2012), or characterize gaze leading abilities in autism spectrum disorders (Dratsch et al., 2013). Edwards, Stephenson, Dalmaso and Bayliss (2015) recently demonstrated that after leading the gaze of an individual, the leader's attention is drawn towards the eyes of the follower, thus favoring the coordination of joint attention between parties. 
Closer to the concerns of the present study, Schilbach et al. (2010) demonstrated in a brain imaging study that gaze leading engaged an area of the brain, the ventral striatum, which is involved in hedonic experiences of reward. Furthermore, this increase in neural activity was linked to a feeling of pleasant experience. Pleasantness in this study referred to the experience of having one's gaze followed by another person and was not a judgement expressing liking for this person. Bayliss et al. (2013) addressed this latter issue in a related study on gaze leading. They conducted series of experiments that used eye-tracking to control faces displayed on a screen. Their results suggested that faces which followed the gaze of participants were preferred over faces that looked in the opposite direction. However, the evidence was weak in that this difference was significant in only one of the two experiments that tested the effect. Additionally, the control conditions used in studies by Schilbach et al. (2010) and Bayliss et al. (2013) relied on faces that produced gaze shifts that were contingent to those of the participant but in a different direction. Therefore, the increase in pleasantness that they observed could alternatively be explained by a negative effect yielded by faces that looked away when participants initiated a gaze shift from the face toward an object. Notwithstanding, these two studies drives us to suspect a link between leading the gaze of another person in a joint attention situation and the propensity to find this person pleasant, that is, liking this person. The present study was designed to assess this hypothesis and complement the previous findings of Schilbach et al. (2010) and Bayliss et al. (2013) by using a different control condition where the avatar would not systematically look away each time the participant initiated gaze movements towards an object of interest.

In addition to pleasantness, gaze behaviors involved in joint attention seem to also influence trustworthiness. Faces that provided predictive gaze cues towards an in-coming target were shown to be judged as more trustworthy than faces looking away from the target (Bayliss, Griffiths, \& Tipper, 2009; Bayliss \& Tipper, 2006). A similar trend has been reported for gaze leading. In a series of experiment conducted by Dalmaso, Edwards and Bayliss (2016), participants rated as more trustworthy faces that followed their gaze compared to faces that looked in the opposite direction. 
However, as before, the observed difference in trustworthiness could be due to a negative effect of looking away rather than to a positive influence of having one's gaze followed. To investigate this issue, our study evaluated trustworthiness in addition to pleasantness. Finally, we also tentatively sought to explore the possible influence of gaze leading on closeness. Closeness is considered to characterize the amount of time and activities shared with another person and the impact one can have on this person's plans (Berscheid, Snyder, \& Omoto, 1989). We put forward the tentative hypothesis that leading the gaze of another individual could promote the impression of sharing an activity with this individual and having an impact on her/his choices. In the present study, pleasantness, trustworthiness and closeness were considered as separate constructs. As emphasized by Schilbach et al. (2010), pleasantness is associated with hedonic feelings and involves rewardrelated cortical networks. By contrast, trustworthiness has been linked to the appraisal of potential threat with brain imaging studies showing increased activity in the amygdala in response to untrustworthy faces (Winston, Strange, O’Doherty, \& Dolan, 2002). Finally, closeness qualifies the interpersonal relationship someone has with another individual and not the individual per se.

As stated above, previous studies have shown that individuals who follow one's gaze are found more pleasant and trustworthy than individuals who look away (Bayliss et al., 2013; Dalmaso et al., 2016). In these studies, the faces that followed gaze were always different from the faces that did not. Our goal was to extend these experiments by testing whether the same individual could be judged more favorably when s/he engaged in gaze following. The present study thus aimed at measuring changes in how individuals were appraised due to them switching to a gaze following behavior. Moreover, we sought to test whether such changes would occur to the same extent in individuals who were initially judged relatively less favorably compared to individuals initially judged more favorably. The experiment was thus performed with two avatars that were physically different, one being more appealing than the other. In previous experiments on gaze leading, participants were instructed to follow a predefined sequence of gaze fixations and gaze shifts. Although these procedures guaranteed experimental controllability, they came at the cost of ecological validity and realism. We 
opted for an experimental setup that enabled participants to have more freedom of movement, thus enhancing ecological validity.

To achieve our goal, we designed advanced versions of avatars endowed with the ability to follow the user's gaze. In previous attempts to draw a connection between gaze leading and pleasantness or trustworthiness (Bayliss et al., 2013; Dalmaso et al., 2016; Schilbach et al., 2010), researchers relied on static human faces that could only move their eyes in pre-defined directions. We developed a system that enabled avatars to simulate naturalistic gaze movements using their eyes and head in a 3D environment. The avatars could follow the gaze of the user in every direction so long as the user was looking at the screen. To further enhance the ecological aspect of the experimental setup, the avatars were based on 3D models of real individuals. Additionally, contrasting with Bayliss et al. (2013) and Dalmaso et al. (2016), we did not limit the comparison of gaze following movements to gaze movements in the opposite direction. In our control condition, gaze movements were independent of the user, that is, they were based on pre-recorded real gaze movements generated by a naïve individual.

Participants were administered a task where they had to look at objects displayed in front of them and decide which one they preferred. While doing so, a human avatar facing them was also attending to the same objects, thus creating a joint attention situation involving the participants. The avatar would either follow the gaze of the participants or explore the objects independently from the participants in the control condition. To extend the validity of our study so that it would not be too contingent on the physical appearance of a single human avatar, the experimental manipulation was applied to two distinct avatars with different degrees of appeal. We report two experiments in the following sections. First, a preliminary experiment is described where the degree of appeal of the two avatars were assessed. Second, we present the main experiment where the avatars were compared between two conditions, that is, when the avatar followed the gaze of participants versus when the avatars moved their gaze autonomously. 


\section{Preliminary experiment}

A person's face physical appearance is known to influence personality judgements made by others. Research studies have identified the baby-faced versus mature-faced dimension as a strong predictor of the personality traits attributed to people (McArthur \& Berry, 1987; Oosterhof \& Todorov, 2008; Zebrowitz \& McDonald, 1991). For instance, legal outcomes tend to be more favorable for babyfaced over more mature-faced individuals in court (Zebrowitz \& McDonald, 1991). Oosterhof and Todorov (2008) showed that baby-like faces are considered less dominant and aggressive. The faces of the two avatars that we designed differed in features associated with face maturity. One of the avatars had larger jawbones, thinner cheeks and more prominent cheekbones than the other. A reasonable hypothesis was therefore that the former would be judged as less appealing than the latter.

\subsection{Method}

\subsubsection{Participants}

Twenty healthy adult individuals ( 10 women and 10 men) were asked to rate videos of the avatars. Their ages ranged from 23 to 49 years with a mean of $30.8(S E=1.6)$.

\subsubsection{Material}

Two male avatars were designed with the Blender 3D software suite (www.blender.org). Their 3D human avatars were modeled from photographs of real individuals using manual graphic design procedures. The avatars' models were similar in terms of gender, age group and ethnicity. They however had distinct features with regard to the baby-faced versus mature-faced dimension (Figure 1). The 3D models of the human avatars were imported into a virtual animation platform called MARC (Multimodal Affective and Reactive Characters). MARC is a toolkit featuring real-time high quality rendering and animation of virtual humans (Courgeon \& Clavel, 2013). Each avatar was displayed on the screen in a virtual scene that included three pictures of items placed on an intermediate plane between the avatar and the participant (see Figure 1). These items were located 
below the head of the avatar. One item was on the left side of the avatar, another on the right and a third in front of the avatar. This layout enabled participants to easily distinguish which object the avatar was staring at. The avatar combined head and eye movements to change their gaze orientation. The angles for neck and eye rotations were computed on the basis of polynomial equations derived from a set of calibration values according to the technical procedure detailed in (Courgeon et al., 2014). It produced no other movements. A 10 seconds long video was produced for each one of the two avatars. In these videos, the avatar was positioned face-to-face with the viewer. The avatar performed two consecutive gazing patterns where it looked back and forth at the three items and then stared at the viewer. The gaze movements of the avatar were created by orienting their focal point of attention towards gaze positions that had been recorded from a real individual using an eye-tracker. This individual was a naïve volunteer who had been asked to explore the virtual scene and then indicate her favorite item among the three that were displayed. We used the procedure explained in Courgeon, Rautureau, Martin and Grynszpan (2014) to orient the focal point of the avatar with respect to the eye-tracking data. We chose to derive the gaze movements of the avatar from the exploration patterns of a real person so as to provide more ecological validity to the stimuli. The same eye-tracking recordings were used in the videos of the two avatars. Hence, the only difference between the two videos was the physical appearance of the avatar. 


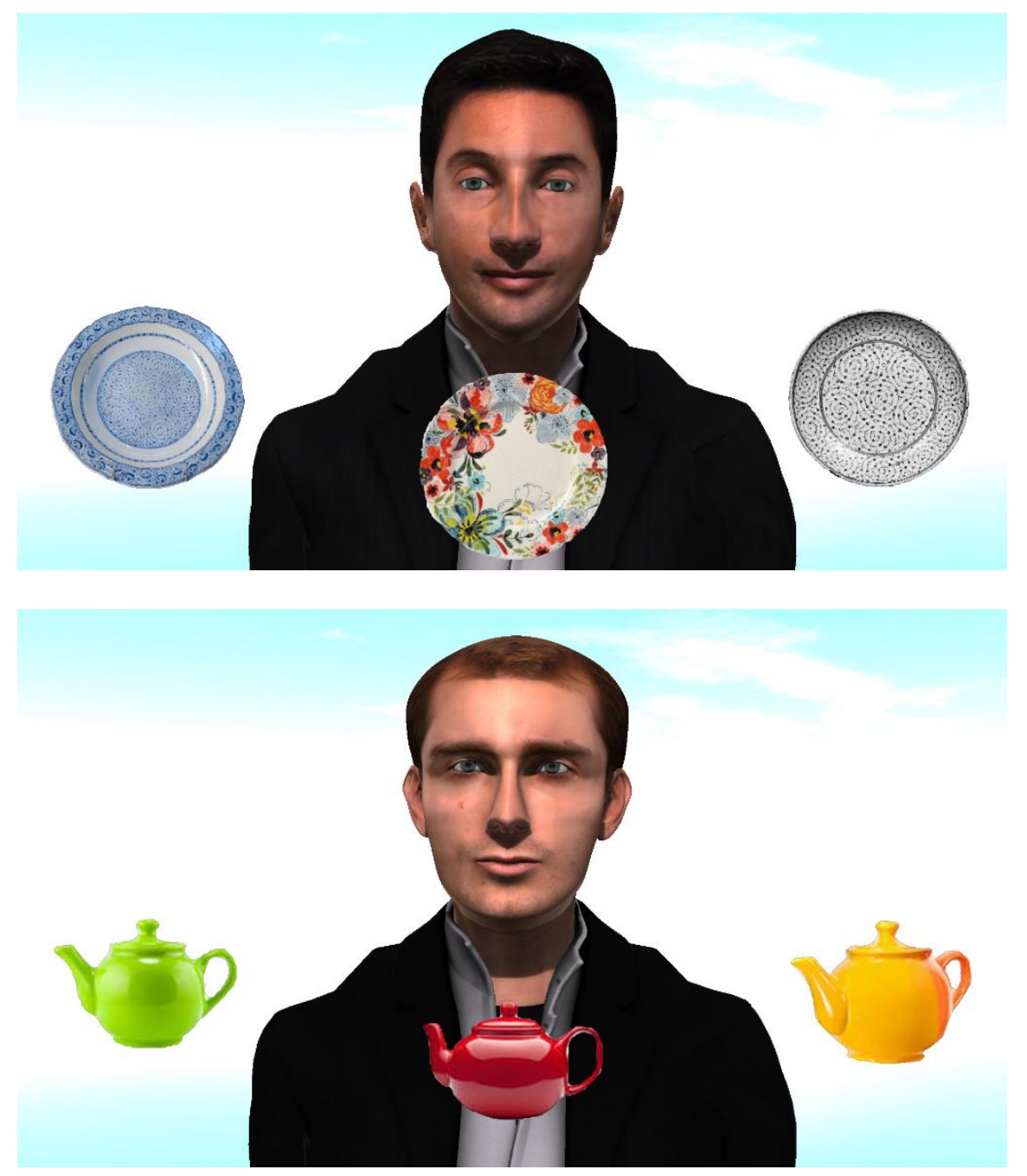

Figure 1: Snapshots of the virtual scenes presented to the participants. Each scene displayed an avatar surrounded by three objects. The two avatars that were compared were named Antony (above) and Mathew (below).

\subsubsection{Procedure}

The two videos were presented to the participants. The order of presentation was randomly counterbalanced across participants. Before they were shown the videos, participants were told that they would have to judge two human characters, who were given commonly used names (Antony for the baby-faced avatar and Mathew for the more mature-faced avatar). After they viewed each video, participants were asked to rate their response to three questions on a visual analogue scale. These questions were the same as those used in the main experiment to assess the effect of gaze following. The first question pertained to the participants' liking of the avatars. The second was intended to examine whether gaze following could also yield trust, as reported in experiments on behavioral mimicry (Maddux, Mullen, \& Galinsky, 2008). The third was meant to evaluate whether participants 
would feel closer to the avatars when the avatars followed their gaze. The three questions were: (1) "To what extent did the character seem pleasant to you?" (2) "To what extent did the character seem trustworthy to you?" (3) "To what extent did you feel close to the character?" The visual analogue scale was a 10 centimeters long line that spread from "Not at all" to "Totally". The responses were measured with a ruler, yielding continuous data from 0 to 10 .

\subsection{Results and discussion}

A one-way multivariate analysis of variance was conducted on scores yielded by the three questions. This multivariate design was used to compare the two avatars. The analyses were performed with the Statistica software application (www.statsoft.com).

A significant difference between the two avatars was found for the first question, which assessed the degree of pleasantness $\left[F(1,19)=11.03 p=0.0036 \eta^{2}=0.37\right]$. The avatar named Antony was considered more pleasant than Mathew (Table 1). There was no significant differences for the two other questions (see Table 1).

Table 1: Means (Standard Errors) of the rating scores yielded by participants for the three questions asked about the two avatars (named Antony and Mathew).

\begin{tabular}{|l|l|l|l|l|}
\cline { 2 - 5 } \multicolumn{1}{c|}{} & Antony & Mathew & $F(1,19)$ & $p$ \\
\hline Pleasantness & $5.25(0.43)$ & $3.56(0.37)$ & 11.03 & $0.0036^{*}$ \\
\hline Trustworthiness & $4.95(0.45)$ & $4.02(0.39)$ & 3.26 & 0.09 \\
\hline Closeness & $4.03(0.49)$ & $2.75(0.46)$ & 3.58 & 0.07 \\
\hline
\end{tabular}

$* p<0.05$

Based on these results, we concluded that the avatar named Antony was more appealing than Mathew. Note should be taken that the average ratings of pleasantness were higher than the middle of the scale for Antony and lower than the middle for Mathew. The higher ratings in pleasantness attributed to Antony are consistent with the literature on personality traits attributed to baby-faced individuals, who are generally regarded as less dominant (McArthur \& Berry, 1987; Oosterhof \& 
Todorov, 2008; Zebrowitz \& McDonald, 1991). The trustworthiness dimension is considered to be orthogonal to the dominance dimension in interpersonal perception and is thus weakly influence by baby-faced appearance (Oosterhof \& Todorov, 2008). The lack of differences in trustworthiness between the two avatars is therefore not surprising. Given that the link between participants and avatars was not manipulated in this experiment, closeness was not expected to vary as it pertains to interpersonal relationships and not to individuals per se. The next experiment was designed with the same two avatars so that the effect of gaze following could be tested on avatars that yielded different degrees of appeal.

\section{Main experiment}

As in the study of Bayliss et al. (2013), the present experiment assessed the impact of gaze following on how the avatars were evaluated when participants were exposed to them in a task-irrelevant manner. Participants had to scan a set of objects and select their favorite one while the avatars were facing them, but they were not explicitly required to direct the gaze orientation of the avatars.

\subsection{Method}

\subsubsection{Participants}

Nineteen participants (10 women and 9 men) were administered the task. Data reported in Schilbach et al. (2010) when comparing pleasantness ratings [Cohen's $d=0.868$ ] were used to perform a power analysis with the G*Power application (Faul, Erdfelder, Lang, \& Buchner, 2007). The power was set at 0.9 and the significance threshold at 0.05 . According to this a prior analysis, the sample size had to be greater than 16. None of the participants participated in the preliminary experiment. Their ages ranged from 19 to 50 years with a mean of $29.9(S E=2.2)$. They were all fluent in French. They were free of any known history of psychiatric or neurologic disorders. They had no visual deficits and did not need to wear glasses. Students or former students in the field of psychology were excluded. These criteria were verified during an individual interview conducted with every participant. Psychiatric disorders were scanned using the Mini International Neuropsychiatric Interview 
(Lecrubier et al., 1997), which is classically employed in clinical settings. As depressive conditions could potentially influence participants' liking experience, mood disorders were specifically scrutinized by administrating additional psychometric questionnaires, that is, the Hamilton Depression Rating Scale (Hamilton, 1960) and the Beck Depression Inventory (Beck, Steer, \& Carbin, 1988). The clinical assessments were performed under the supervision of a professional psychiatrist. The entire experiment took place in the premises of a hospital. Participants received $15 €$ as a compensation for completing the experiment. This research was reviewed and approved by the local ethics committee. An informed consent was obtained from each participant.

\subsubsection{Material}

Participants were seated in front of a computer screen that displayed the same virtual scene as described in the preliminary experiment, that is, one avatar and three items placed in front of it, below its head (Figure 1). The two avatars tested in the preliminary experiment were alternately used. The computer was equipped with an eye-tracker, model X2-60 from Tobii (www.tobii.com). This eye-tracker remotely detects the direction of the eyes without the need for a chin rest or a helmet. Participants could therefore move their eyes and head freely. The sampling rate of the eyetracker was $60 \mathrm{~Hz}$, its accuracy was of $0.4^{\circ}$. The screen size was $380 \times 215 \mathrm{~mm}^{2}$ with a resolution of $1920 \times 1080$ pixels. Participants were placed at an approximate $60-65 \mathrm{~cm}$ distance from the screen, in compliance with the eye-tracker's specifications. The dimensions in visual angles of the avatar's head were $7.7^{\circ} \times 10.1^{\circ}$ and the items $s^{\prime}$ dimensions were $6.9^{\circ} \times 6.9^{\circ}$. One item was placed $11.7^{\circ}$ rightwards from the center of the screen, another one $11.7^{\circ}$ leftwards for the center and the third one $5.9^{\circ}$ below the center. Using the Python programming language and the Tobii Software Development Kit, we developed software that enabled the avatar to follow the gaze of the participant via the eyetracker by sending Behavior Markup Language (Kopp et al., 2006) messages to the MARC platform in real-time. More details regarding the method used to implement gaze following by an avatar via an eye-tracker can be found in (Courgeon et al., 2014). The participant's gaze positions on the screen were averaged with a sliding time window of 100 milliseconds to minimize potential erratic 
movements of the avatar induced by micro-saccades and eye-tracking imprecisions. The experimenter could monitor the performances of the eye-tracker throughout the experiment on a graphic interface that was displayed on a second screen placed behind the participant. A latency of two seconds was introduced between changes in the participant's gaze position and the ensuing redirection of the avatar's gaze orientation. This latency was meant to create a contingency between the participant's gaze and the movement of the avatar that would not be too obvious and potentially irritating. In an experiment by Pfeiffer et al. (2012) where the latency of gaze following movements by an avatar was varied, participants were asked to rate their feeling of how related the reaction of the avatar was to their own behavior. Their ratings for a latency of two seconds were on average in the middle of the Likert scale, that is, in-between "rather related" and "rather unrelated" (see Figure $2 \mathrm{~B}$ on page 5 of their article). We thus devised that the latency of two seconds that we chose would yield an ambiguous awareness of the contingent reactions yielded by the avatars. We also wanted to avoid a situation where each time the participant looked at the avatar, the latter would be immediately staring back at her/him.

There were two modes of behavior for the avatar displayed on the screen. It would either follow the gaze of the participant as explained above (gaze following mode) or its behavior would be autonomous (autonomous gaze mode). To generate the autonomous gaze behavior, we used recordings of two real individuals' gaze as described in the preliminary experiment. The recordings of these two individuals were stored in a common database used by our software so that the avatars would reproduce gazing patterns originating from either one of them. This guaranteed that the autonomous gazing behavior of the avatars would not be too much influenced by the specific gazing behavior of a particular individual.

\subsubsection{Procedure}

The experiment started with a standard calibration procedure for the eye-tracker, where the participant had to fixate five points appearing in the corners and at the center of the graphic display. 
Before starting the experiment, participants were explained that they would be viewing objects and that an avatar facing them would also be observing these objects. They were told that their task would be to choose their preferred object. They were also asked to pay attention to the avatar, because they would have to answer questions about it.

Each participant was then administered four blocks of 10 trials each. Each trial began with a fixation cross displayed for two seconds. The participant was then exposed during 20 seconds to the same virtual scene as described in the preliminary experiment, where an avatar was facing the participant and three items were located in an intermediate plane between the avatar and the participant. The items were consumer goods such as cellular phones, watches, laptop computers, dishes, small furniture etc. They belonged to an image database that was constructed for this experiment. As participants' task was to select their favorite item, we chose attractive consumer goods that were expected to arise interest. The items displayed changed with every trial. The items that appeared together during a single trial belong to the same category of objects, that is, they were all watches or all phones etc. After the trial, the participant was to answer a forced choice question asking which was her/his favorite item among the three that had just been displayed.

The gaze behavior mode of the avatar and its identity (Antony or Mathew) were consistent in all the trials of a block. At the end of each block, the participant had to rate her/his response to the same three questions as in the preliminary experiment regarding the pleasantness, the trustworthiness and the closeness of the avatar. The same visual analogue scale as in the preliminary experiment was again used. The identity of the avatar (Antony or Mathew) changed with every new block. Each avatar therefore appeared in two different blocks. The gaze behavior mode of each avatar differed in the two blocks where it appeared, that is, the avatar would either be following the gaze of the participant or it would be functioning autonomously. Each block thus presented a unique combination of the identity of the avatar and the gaze behavior mode. In the blocks where the avatar adopted the autonomous gaze mode, there were enough recordings of real gaze movements so that 
the gazing patterns would be different in every trial. The order of the blocks was counterbalanced across participants, that is, the order of the avatar's identity (Antony vs. Mathew) was counterbalanced for each gaze behavior mode (Gaze following vs. Autonomous gaze) and the order of the gaze behavior mode was counterbalanced for each avatar identity.

\subsection{Results}

A two way multivariate analysis of variance (MANOVA) design was applied on rating scores yielded by the three questions asked after each block. The two factors of the MANOVA were the avatar identity (Antony vs. Mathew) and the behavior mode (Gaze following vs. Autonomous gaze). The statistical computations were conducted with the Statistica software application (www.statsoft.com).

For the question related to the pleasantness of the avatar, there was a main effect of the avatar identity $\left[F(1,18)=10.30 p=0.0049 \eta^{2}=0.36\right]$ and of the behavior mode $[F(1,18)=5.19 p=0.0352$ $\eta^{2}=0.22$ ] (see Figure 2). Participants considered that the avatar named Antony was more pleasant than the avatar named Mathew [Antony: mean $=6.96(\mathrm{SE}=0.41)$; Mathew: mean $=5.61(\mathrm{SE}=0.46)$ ]. The gaze following mode was rated as more pleasant than the autonomous gaze mode [Gaze following: mean $=6.58(\mathrm{SE}=0.45)$; Autonomous gaze: mean $=5.99(\mathrm{SE}=0.42)]$. There was no interaction between the two factors $[F(1,18)=0.20 p=0.66]$. 


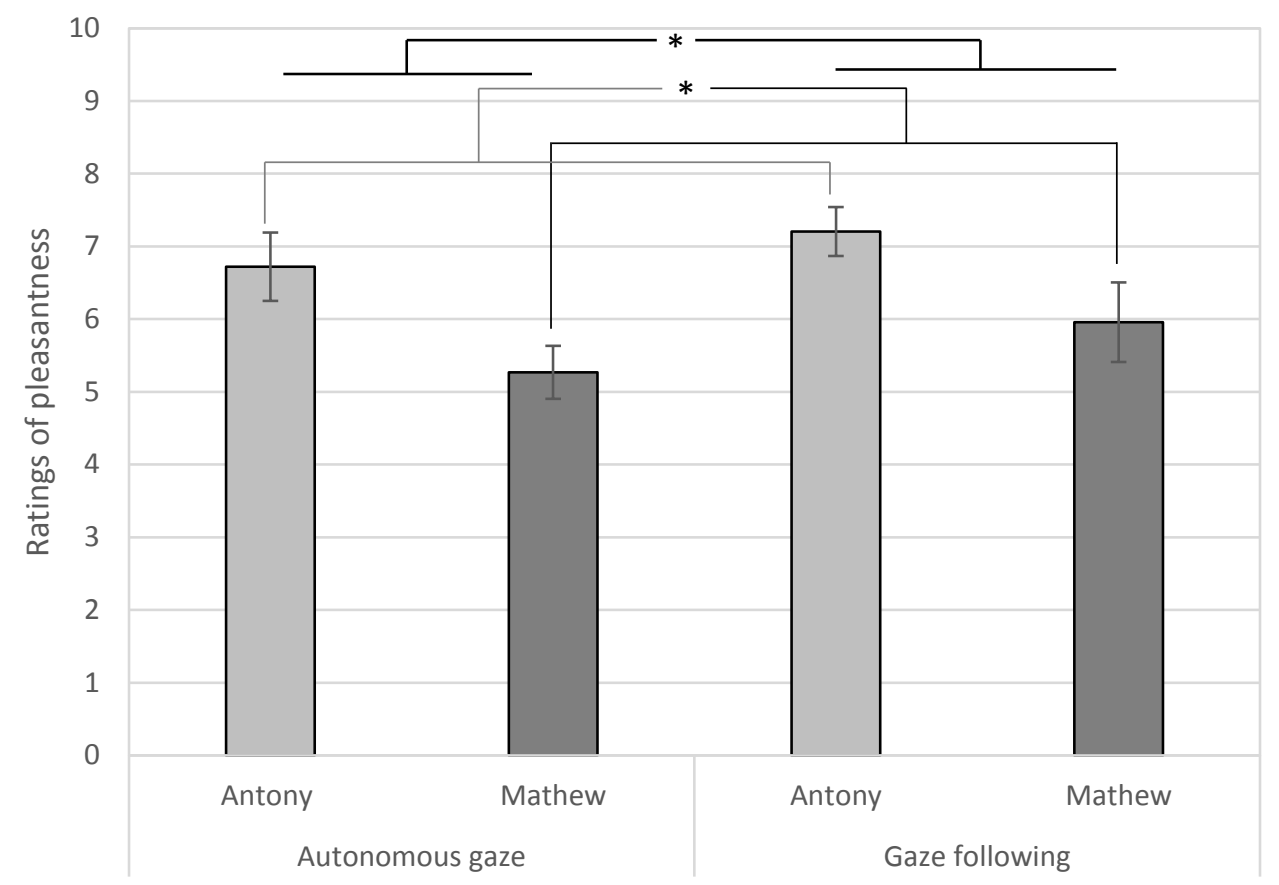

Figure 2: Mean ratings with standard errors for the avatars in the four blocks. The data in the autonomous gaze mode is displayed on the left, while the data in the gaze following mode is displayed on the right. The two avatars were given two different names, that is, Antony and Mathew.

$* p<0.05$

For the question related to trustworthiness, there was only a main effect of the avatar identity $\left[F(1,18)=5.10 p=0.0366 \eta^{2}=0.22\right]$. Participants rated Antony as more trustworthy than Mathew [Antony: mean $=6.62(S E=0.43) ;$ Mathew: mean $=5.54(S E=0.50)]$. The main effect of behavior mode was not significant $[F(1,18)=2.16 p=0.16]$, nor was the interaction between the two factors $[F(1,18)=1.34 p=0.26]$

For the question related to closeness, the main effect of the avatar identity was insignificant yet close to the significance threshold $[F(1,18)=4.22 p=0.055]$. There was no significant main effect of behavior mode $[F(1,18)=3.74 p=0.07]$, nor was the interaction significant $[F(1,18)=1.96 p=0.18]$. Descriptive statistics for the three questions are provided in Table2. 
Table 2: Means (Standard Errors) of rating scores provided by participants for the two avatars (named Antony and Mathew) in the two behavior modes (autonomous gaze vs. gaze following)

\begin{tabular}{|l|l|l|l|l|}
\cline { 2 - 5 } \multicolumn{1}{c|}{} & \multicolumn{2}{c|}{ Autonomous gaze } & \multicolumn{2}{c|}{ Gaze following } \\
\cline { 2 - 5 } \multicolumn{1}{c|}{} & Antony & Mathew & Antony & Mathew \\
\hline Pleasantness & $6.72(0.47)$ & $5.27(0.36)$ & $7.21(0.34)$ & $5.96(0.55)$ \\
\hline Trustworthiness & $6.49(0.44)$ & $5.12(0.44)$ & $6.76(0.42)$ & $5.96(0.55)$ \\
\hline Closeness & $5.41(0.53)$ & $4.39(0.51)$ & $5.99(0.48)$ & $5.64(0.62)$ \\
\hline
\end{tabular}

\section{Discussion}

The results of the main experiment indicate an increase in liking when the avatars were following the gaze of participants. This outcome supports our hypothesis of a relationship between liking and gaze leading. The lack of interaction between the gazing behavior and the identity of the avatars suggests that this effect was independent of the physical appearance of the avatars. The pleasantness attributed to each avatar was assessed independently of how they gazed in the preliminary experiment. The superior appeal of one (Antony) over the other (Mathew) was confirmed in the main experiment, as revealed by the main effect of the avatar's identity. Notwithstanding, the two avatars were rated as more pleasant when they followed the gaze of the participant, as opposed to when they looked at objects in a way that was unconnected to the participant. One could argue that the gaze-following effect on pleasantness ratings could have been driven by mere behavioral contingencies between the eye movements of the participant and changes in the avatar's gaze orientation. The study by Bayliss et al. (2013) however advocates against this thesis as they showed an effect of gaze-following when it was compared to a condition where the gaze of the participant and the gaze of the avatar were contingent but moved in opposite directions. The outcome of our study provides evidence for the position held in previous studies (Bayliss et al., 2013; Schilbach et al., 2010) that gaze leading induces a hedonic feeling and fosters liking. It extends these findings by showing that having one's gaze followed by an individual changes the level of liking for this 
individual, even when the baseline level of liking is relatively low. Moreover, the control condition used here discards the alternative possible explanation that the increase in liking found in previous studies was not due to gaze following but to a negative effect of averted gaze.

Participants were asked to judge the avatars not only for their pleasantness, but also for their trustworthiness and their closeness. None of the two latter were influenced by the gaze following behavior of the avatar. Participants in the preliminary experiment did not rate one avatar as more trustworthy or than the other, while participants in the main experiment found that the avatar that they considered the most pleasant was also more trustworthy. The perception of closeness followed the same trend. The reason may be that participants were exposed for a longer period of time to the two avatars in the main experiment. An alternative hypothesis is that the gaze following behavior displayed by the avatars could have exacerbated the difference in how they were perceived by the participants. Noteworthy is the fact that pleasantness ratings increased in the main experiment compared to the preliminary experiment, even when the avatars' gaze behavior was autonomous. A possible explanation is that longer exposition time in the main experiment induced habituation that favored positive feelings towards the avatars. Mere exposure to faces is known to enhance attitude towards them (Zajonc, 1968).

Neither trustworthiness nor closeness were influence by the gaze following behavior of the avatars. The lack of effect on trustworthiness apparently contrasts with the study by Dalmaso, Edwards and Bayliss (2016). However, in their experiment, participants were to either produce saccades or antisaccades depending on the color of a target and the faces that subsequently followed the gaze of the participants were consistently associated with the target's color for saccades. Therefore, although these faces looked at the target after the participant did, they were nevertheless informative of the type of eye movement expected from the participant, that is, a saccade. As emphasized by Bayliss and Tipper (2006), the trustworthiness of faces is mediated by the informative value of their gaze cues. In our study, the gaze following behavior of the avatars was task irrelevant and did not provide 
any specific information to participants. The non-informative value of the gaze following behavior provides a plausible reason for it failing to influence trustworthiness. As mentioned above, the feeling of closeness was not altered by the gaze following behavior of the avatars. This can be interpreted as a lack of recognition by the participants that they_were sharing an experience with the avatars and that they could have an influence on the avatars' choices. However, this interpretation should be considered with caution as the effect of gaze following on closeness was not far from the significance threshold $(p=0.07)$.

Edwards et al. (2015) suggest that the rewarding experience of causing another person to re-orient attention in the same direction as ours may be comparable to the detection of being imitated. Following this line of thought, the relationship between gaze leading in joint attention and liking echoes similar findings related to interpersonal imitative behaviors, notably mimicry and what is known as the chameleon effect (Chartrand \& Bargh, 1999). The chameleon effect refers to our natural unconscious tendency to mimic the mannerisms of others (e.g. rubbing one's face or shaking one's foot) during social interactions. It has been shown to foster smoothness in the interaction and increase liking between social partners (Chartrand \& Bargh, 1999; Lakin, Jefferis, Cheng, \& Chartrand, 2003). A main dissimilitude however between the chameleon effect and the effect reported here is that the chameleon effect usually pertains to behaviors that have no functional relevance in human communication. Those behaviors are described as nonconscious mannerisms that go with the ongoing conversation but are detached from it, that is, they do not convey any meaningful message. By contrast, as explained in the introduction, gaze following is an important building block of joint attention and thus holds a highly functional role in human communication. The present study therefore suggests that the relationship between mimicry and liking could extend to non-verbal communicative behaviors also.

When comparing the influence of gaze following on liking with the chameleon effect, we do not intend to draw a strict equivalence between mimicry and the gaze coupling behaviors that occur 
during joint attention. Rather, we seek to highlight mechanisms that may be common to different forms of imitation recognition. In the chameleon effect, individuals are not aware of being mimicked. But in the case of gaze leading however, explicit recognition of being followed may happen. For instance, in our experiment, the avatars followed the gaze of participants in a task-irrelevant manner. After the experiment, participants we debriefed during an informal interview based on a set of questions that were meant to probe their understanding of the experiment's goals. Twelve participants declared that they had noticed that, at some point in time, the avatars followed their gaze. None however mentioned that their awareness of being followed influenced how they rated the avatars. According to Nadel (2002), there are several levels of imitation recognition that extend from implicit perception-action coupling to the explicit awareness of another person's intention to imitate. Recognizing that one's gaze is being followed could also vary along this continuum. Future research could investigate how explicit recognition modulates the liking effect yielded by gaze following.

The results of the present study suggest that liking was enhanced by gaze following, even when the gaze-follower was initially less favorably judged. Stel et al. (2010) found that people who were disliked a priori did not benefit from the positive effect of mimicry on liking. However, these authors used cover stories to induce participants to dislike target individuals. Liking was thus guided by reflexive and explicit reasoning. By contrast, in our study, initial liking varied on the basis of physical appearance, which elicits implicit and automatic mechanisms of personality trait attribution. The impact of a priori contextual factors may depend on whether they involve implicit or explicit cognitive control of liking. More research on the influence of a priori moderators of liking would be insightful to better understand the cognitive processes associated with gaze following.

The current study has several limitations. First, it was an exploratory study with a relatively small sample, although the sample size was comparable to those used in previous studies on the topic (Bayliss et al., 2013; Edwards et al., 2015; Schilbach et al., 2010). Reproducing similar 
experimentations with more participants could amplify the power of the statistical tests performed and yield effects on trustworthiness or closeness that were not detected here. Second, the two avatars used in the experiments were both males. This leaves open the possibility that the outcomes would have been different with female avatars. Moreover, due to the limited number of participants, we did not differentiate between the answers of male and female participants, who could diverge in their ratings of the avatars. Third, the gaze following latency was fixed to two seconds in the current experiment. Future studies are warranted to assess the effect of latency on liking. Finally, the study relied on avatars because they enabled enhanced controllability that guarantied the effectiveness of gaze following behaviors. Although they were design to be very realistic, they still could not fool participants into believing that they were real human beings. Notwithstanding, the present study yielded outcomes that should be of relevance to the community interested in the design of embodied conversational agents (ECA), which are virtual agents that simulate some features of faceto-face multimodal human conversation (Cassell, 2000). The chameleon effect has been implemented in virtual agents and has shown to improve their communicative performances (Bailenson \& Yee, 2005). Likewise, endowing such agents with the ability to follow the gaze of users should enhance their pleasantness and therefore their acceptability.

\section{Acknowledgements}

This work was supported by a grant from L'Agence Nationale de la Recherche (project \#ANR 12 SAMA 011 01, coordinator: P. Fossati). The authors wish to thank Nicolas Vienney for experimental assistance.

\section{References}

Bailenson, J. N., \& Yee, N. (2005). Digital Chameleons Automatic Assimilation of Nonverbal Gestures in Immersive Virtual Environments. Psychological Science, 16(10), 814-819. https://doi.org/10.1111/j.1467-9280.2005.01619.x 
Bailly, G., Raidt, S., \& Elisei, F. (2010). Gaze, conversational agents and face-to-face communication. Speech Communication, 52(6), 598-612. https://doi.org/10.1016/j.specom.2010.02.015

Baron-Cohen, S. (1997). Mindblindness: An Essay on Autism and Theory of Mind. Cambridge, MA, USA: MIT Press.

Baron-Cohen, S., Campbell, R., Karmiloff-Smith, A., Grant, J., \& Walker, J. (1995). Are children with autism blind to the mentalistic significance of the eyes? British Journal of Developmental Psychology, 13(4), 379-398. https://doi.org/10.1111/j.2044-835X.1995.tb00687.x

Baron-Cohen, S., Wheelwright, S., \& Jolliffe, T. (1997). Is there a« language of the eyes »? Evidence from normal adults, and adults with autism or Asperger syndrome. Visual Cognition, 4(3), 311-331. https://doi.org/10.1080/713756761

Bayliss, A. P., Griffiths, D., \& Tipper, S. P. (2009). Predictive gaze cues affect face evaluations: The effect of facial emotion. European Journal of Cognitive Psychology, 21(7), 1072-1084. https://doi.org/10.1080/09541440802553490

Bayliss, A. P., Murphy, E., Naughtin, C. K., Kritikos, A., Schilbach, L., \& Becker, S. I. (2013). « Gaze leading »: initiating simulated joint attention influences eye movements and choice behavior. Journal of Experimental Psychology. General, 142(1), 76-92. https://doi.org/10.1037/a0029286

Bayliss, A. P., \& Tipper, S. P. (2006). Predictive gaze cues and personality judgments: Should eye trust you? Psychological Science, 17(6), 514-520. https://doi.org/10.1111/j.14679280.2006.01737.x

Beck, A. T., Steer, R. A., \& Carbin, M. G. (1988). Psychometric properties of the Beck Depression Inventory: Twenty-five years of evaluation. Clinical Psychology Review, 8(1), 77-100. https://doi.org/10.1016/0272-7358(88)90050-5

Berscheid, E., Snyder, M., \& Omoto, A. M. (1989). The Relationship Closeness Inventory: Assessing the closeness of interpersonal relationships. Journal of Personality and Social Psychology, 57(5), 792-807. https://doi.org/10.1037/0022-3514.57.5.792 
Cassell, J. (2000). Embodied Conversational Agents. Cambridge, MA, USA: MIT Press.

Charman, T. (2003). Why is joint attention a pivotal skill in autism? Philosophical Transactions of the Royal Society of London. Series B, Biological Sciences, 358(1430), 315-324. https://doi.org/10.1098/rstb.2002.1199

Chartrand, T. L., \& Bargh, J. A. (1999). The chameleon effect: the perception-behavior link and social interaction. Journal of Personality and Social Psychology, 76(6), 893-910. https://doi.org/10.1037/0022-3514.76.6.893

Courgeon, M., \& Clavel, C. (2013). MARC: a framework that features emotion models for facial animation during human-computer interaction. Journal on Multimodal User Interfaces, 7(4), 311-319. https://doi.org/10.1007/s12193-013-0124-1

Courgeon, M., Rautureau, G., Martin, J.-C., \& Grynszpan, O. (2014). Joint Attention Simulation Using Eye-Tracking and Virtual Humans. IEEE Transactions on Affective Computing, 5(3), 238-250. https://doi.org/10.1109/TAFFC.2014.2335740

Dalmaso, M., Gareth, S., \& Bayliss, A. P. (2016). Re-encountering individuals who previously engaged in joint gaze modulates subsequent gaze cueing. Journal of Experimental Psychology: Learning, Memory, and Cognition, 42(2), 271-284. https://doi.org/10.1037/xlm0000159

Dratsch, T., Schwartz, C., Yanev, K., Schilbach, L., Vogeley, K., \& Bente, G. (2013). Getting a grip on social gaze: control over others' gaze helps gaze detection in high-functioning autism. Journal of Autism and Developmental Disorders, 43(2), 286-300. https://doi.org/10.1007/s10803012-1569-x

Driver, J. (1999). Gaze Perception Triggers Reflexive Visuospatial Orienting. Visual Cognition, 6(5), 509 -540. https://doi.org/10.1080/135062899394920

Edwards, S. G., Stephenson, L. J., Dalmaso, M., \& Bayliss, A. P. (2015). Social orienting in gaze leading: a mechanism for shared attention. Proc. R. Soc. B, 282(1812), 20151141. https://doi.org/10.1098/rspb.2015.1141 
Emery, N. J. (2000). The eyes have it: the neuroethology, function and evolution of social gaze. Neuroscience and Biobehavioral Reviews, 24(6), 581-604. https://doi.org/10.1016/S01497634(00)00025-7

Faul, F., Erdfelder, E., Lang, A.-G., \& Buchner, A. (2007). G*Power 3: A flexible statistical power analysis program for the social, behavioral, and biomedical sciences. Behavior Research Methods, 39(2), 175-191. https://doi.org/10.3758/BF03193146

Frischen, A., Bayliss, A. P., \& Tipper, S. P. (2007). Gaze cueing of attention: visual attention, social cognition, and individual differences. Psychological Bulletin, 133(4), 694-724. https://doi.org/10.1037/0033-2909.133.4.694

Haith, M. M., Bergman, T., \& Moore, M. J. (1977). Eye contact and face scanning in early infancy. Science (New York, N.Y.), 198(4319), 853-855. https://doi.org/10.1126/science.918670 Hamilton, M. (1960). A RATING SCALE FOR DEPRESSION. Journal of Neurology, Neurosurgery, and Psychiatry, 23(1), 56-62.

Hoekstra, A., Prendinger, H., Bee, N., Heylen, D., \& Ishizuka, M. (2007). Highly Realistic 3D Presentation Agents with Visual Attention Capability. In A. Butz, B. Fisher, A. Krüger, P. Olivier, \& S. Owada (Ed.), Smart Graphics (p. 73-84). Springer Berlin Heidelberg.

Kim, K., \& Mundy, P. (2012). Joint Attention, Social-Cognition, and Recognition Memory in Adults. Frontiers in Human Neuroscience, 6. https://doi.org/10.3389/fnhum.2012.00172

Kobayashi, H., \& Kohshima, S. (2001). Unique morphology of the human eye and its adaptive meaning: comparative studies on external morphology of the primate eye. Journal of Human Evolution, 40(5), 419-435. https://doi.org/10.1006/jhev.2001.0468

Kopp, S., Krenn, B., Marsella, S., Marshall, A. N., Pelachaud, C., Pirker, H., ... Vilhjálmsson, H. (2006). Towards a Common Framework for Multimodal Generation: The Behavior Markup Language. In J. Gratch, M. Young, R. Aylett, D. Ballin, \& P. Olivier (Ed.), Intelligent Virtual Agents (p. 205217). Springer Berlin Heidelberg. https://doi.org/10.1007/11821830_17 
Lachat, F., Conty, L., Hugueville, L., \& George, N. (2012). Gaze Cueing Effect in a Face-to-Face Situation. Journal of Nonverbal Behavior, 36(3), 177-190. https://doi.org/10.1007/s10919012-0133-x

Lakin, J. L., Jefferis, V. E., Cheng, C. M., \& Chartrand, T. L. (2003). The Chameleon Effect as Social Glue: Evidence for the Evolutionary Significance of Nonconscious Mimicry. Journal of Nonverbal Behavior, 27(3), 145-162. https://doi.org/10.1023/A:1025389814290

Lecrubier, Y., Sheehan, D., Weiller, E., Amorim, P., Bonora, I., Harnett Sheehan, K., ... Dunbar, G. (1997). The Mini International Neuropsychiatric Interview (MINI). A short diagnostic structured interview: reliability and validity according to the CIDI. European Psychiatry, 12(5), 224-231. https://doi.org/10.1016/S0924-9338(97)83296-8

Maddux, W. W., Mullen, E., \& Galinsky, A. D. (2008). Chameleons bake bigger pies and take bigger pieces: Strategic behavioral mimicry facilitates negotiation outcomes. Journal of Experimental Social Psychology, 44(2), 461-468. https://doi.org/10.1016/j.jesp.2007.02.003

McArthur, L. Z., \& Berry, D. S. (1987). Cross-Cultural Agreement in Perceptions of Babyfaced Adults. Journal of Cross-Cultural Psychology, 18(2), 165-192. https://doi.org/10.1177/0022002187018002003

Mundy, P., \& Newell, L. (2007). Attention, Joint Attention, and Social Cognition. Current Directions in Psychological Science (Wiley-Blackwell), 16(5), 269-274. https://doi.org/10.1111/j.14678721.2007.00518.x

Nadel, J. (2002). Imitation and imitation recognition: Functional use in preverbal infants and nonverbal children with autism. In The imitative mind: Development, evolution, and brain bases (eds AN Meltzoff, W Prinz, p. 42-62). Cambridge, UK: Cambridge University Press. Oberwelland, E., Schilbach, L., Barisic, I., Krall, S. C., Vogeley, K., Fink, G. R., ... Schulte-Rüther, M. (2016). Look into my eyes: Investigating joint attention using interactive eye-tracking and fMRI in a developmental sample. Neurolmage, 130, 248-260.

https://doi.org/10.1016/j.neuroimage.2016.02.026 
Oosterhof, N. N., \& Todorov, A. (2008). The functional basis of face evaluation. Proceedings of the National Academy of Sciences, 105(32), 11087-11092. https://doi.org/10.1073/pnas.0805664105

Peters, C., Asteriadis, S., \& Karpouzis, K. (2010). Investigating shared attention with a virtual agent using a gaze-based interface. Journal on Multimodal User Interfaces, 3(1-2), 119-130. https://doi.org/10.1007/s12193-009-0029-1

Pfeiffer, U. J., Schilbach, L., Jording, M., Timmermans, B., Bente, G., \& Vogeley, K. (2012). Eyes on the mind: investigating the influence of gaze dynamics on the perception of others in real-time social interaction. Frontiers in Psychology, 3, 537. https://doi.org/10.3389/fpsyg.2012.00537

Posner, M. I. (1980). Orienting of attention. The Quarterly Journal of Experimental Psychology, 32(1), 3-25. https://doi.org/10.1080/00335558008248231

Redcay, E., Kleiner, M., \& Saxe, R. (2012). Look at this: the neural correlates of initiating and responding to bids for joint attention. Frontiers in Human Neuroscience, 6, 169. https://doi.org/10.3389/fnhum.2012.00169

Scaife, M., \& Bruner, J. S. (1975). The capacity for joint visual attention in the infant. Nature, 253(5489), 265-266. https://doi.org/10.1038/253265a0

Schilbach, L., Wilms, M., Eickhoff, S. B., Romanzetti, S., Tepest, R., Bente, G., ... Vogeley, K. (2010). Minds made for sharing: initiating joint attention recruits reward-related neurocircuitry. Journal of Cognitive Neuroscience, 22(12), 2702-2715. https://doi.org/10.1162/jocn.2009.21401

Stel, M., Blascovich, J., McCall, C., Mastop, J., van Baaren, R. B., \& Vonk, R. (2010). Mimicking disliked others: Effects of a priori liking on the mimicry-liking link. European Journal of Social Psychology, 40(5), 867-880. https://doi.org/10.1002/ejsp.655

Wang, H., Chignell, M., \& Ishizuka, M. (2006). Empathic tutoring software agents using real-time eye tracking. In Proceedings of the 2006 symposium on Eye tracking research \& applications (p. 73-78). New York, NY, USA: ACM. 
Wilms, M., Schilbach, L., Pfeiffer, U., Bente, G., Fink, G. R., \& Vogeley, K. (2010). It's in your eyesusing gaze-contingent stimuli to create truly interactive paradigms for social cognitive and affective neuroscience. Social cognitive and affective neuroscience, 5(1), 98. https://doi.org/10.1093/scan/nsq024

Winston, J. S., Strange, B. A., O’Doherty, J., \& Dolan, R. J. (2002). Automatic and intentional brain responses during evaluation of trustworthiness of faces. Nature Neuroscience, 5(3), 277-283. https://doi.org/10.1038/nn816

Zajonc, R. B. (1968). Attitudinal effects of mere exposure. Journal of Personality and Social Psychology, 9(2p2), 1. https://doi.org/10.1037/h0025848

Zebrowitz, L. A., \& McDonald, S. M. (1991). The impact of litigants' baby-facedness and attractiveness on adjudications in small claims courts. Law and Human Behavior, 15(6), 603-623.

https://doi.org/10.1007/BF01065855 